\title{
Analytical techniques for deoxynivalenol detection and quantification in wheat destined for the manufacture of commercial products
}

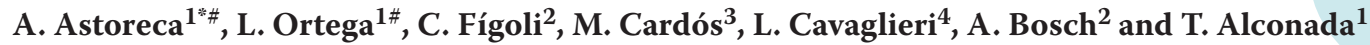 \\ ${ }^{1}$ Centro de Investigación y Desarrollo en Fermentaciones Industriales (CINDEFI), CONICET-Facultad de Ciencia, Exactas, \\ Universidad Nacional de La Plata, calle 47 y 115, B1900ASH La Plata, Argentina; ${ }^{2}$ CINDEFI, CONICET-Facultad de \\ Ciencias Exactas, Universidad Nacional de La Plata, Laboratorio de Bioespectroscopía, calle 47 y 115, B1900ASH La Plata, \\ Argentina; ${ }^{3}$ Molino Campodónico, calle 58 No. 331, B1900BPM La Plata, Argentina; ${ }^{4}$ Departamento de Microbiología \\ e Inmunología, Facultad de Ciencias Exactas, Físico Químicas y Naturales, Universidad Nacional de Río Cuarto, Ruta \\ Nacional 36 Km 601, 5800 Río Cuarto, Córdoba, Argentina; astoreca@biotec.quimica.unlp.edu.ar; \# these authors \\ contributed equally to this work
}

Received: 27 July 2016 / Accepted: 14 October 2016

(c) 2016 Wageningen Academic Publishers

RESEARCH ARTICLE

\begin{abstract}
The concern regarding toxicity from the presence of deoxynivalenol (DON) in wheat that affects both economy and public health leads to the need to find appropriate detection methods for determining the degree of DON contamination in terms of the equipment available and the speed required for obtaining the incidence. The objective of this study was to compare the performance of two alternative analytical techniques for DON quantification for use in the food industry with a reference technique. Samples of wheat and the commercial by-products were analysed by high-performance liquid chromatography (HPLC) with an ultraviolet detector as the reference method and the results compared with those obtained from a rapid lateral-flow immunochromatographic device (Reveal Q+) and of a Fourier-transform-infrared (FTIR) spectroscopy technique. Pearson's correlation coefficient between the HPLC and Reveal-Q+ data (0.45), although significant $(P<0.0003)$, was lower than that obtained between HPLC and the FTIR method $(0.94, P<0.0001)$. Both methods were considered efficient in quantifying DON levels in wheat-flour samples. This study was aimed at assisting the producers in choosing an appropriate tool for the purpose of analysis and upon consideration of the available equipment.
\end{abstract}

Keywords: deoxynivalenol, wheat, high-performance liquid chromatography, Fourier-transform infrared spectroscopy, lateral-flow immunochromatography

\section{Introduction}

Wheat is an essential food source for many people around the world (Shewry, 2009). Special attention is required regarding health-related and commercialisation issues, such as the major threat to public health and agroeconomy posed by Fusarium-toxin contamination. Within this group, deoxynivalenol (DON) is the most relevant mycotoxin for wheat and wheat-derived products due to the toxin's widespread occurrence and the potential impact on animal and human health (Prelusky et al., 1994). The detection of DON is usually a good indicator that other Fusarium toxins are also present in the same substrate (Sobrova et al.,
2010). Since the number of reported DON-contamination incidents has increased during the last ten years, the development and application of various DON-detection methods represents a pressing issue in the food industry (Koch, 2004). In view of the potential danger that DON represents to human and animal health, the European Community set a threshold for this toxin at $1.75 \mu \mathrm{g} / \mathrm{g}$ for unprocessed durum-wheat grains, $0.75 \mu \mathrm{g} / \mathrm{g}$ for cereals (wheat, maize, and barley) for direct human consumption or for flours and dried pastas, $0.50 \mu \mathrm{g} / \mathrm{g}$ for bread, and 0.20 $\mu \mathrm{g} / \mathrm{g}$ for cereal-based foods for infants and young children (EC, 2007). 
The analytical methods most frequently used for the detection and quantitation of these metabolites in wheat and by-products are liquid chromatography and gas chromatography, owing to the precise specificity and high sensitivity of those techniques. Liquid chromatography is a useful tool because of the ability to separate substances with different characteristics (heat-labile, nonvolatile, polar, and nonpolar), thus constituting a reproducible technique for accurate quantitation (Ran et al., 2013). Nevertheless, liquid chromatography is costly, requires highly qualified personnel, and is time-consuming. For these reasons and in response to the demand for rapid screening for mycotoxins - an extensive number of immunological technologies have been developed for quantitation of DON in cereals; and, depending on the purpose of the analysis, different kits have been manufactured in several lateral-flow formats. All of these commercial kits employ a procedure that is particularly well suited for on-site detection and are designed for the analysis of single samples by users having minimal laboratory training (Alldrick et al., 2009). The Reveal-Q+ assay for DON - a single-step lateral-flow immunochromatographic assay based on a competitiveimmunoassay format intended for the quantitative testing of DON in maize, barley, malted barley, oats, and wheat products - is used for routine measurement for DON quantification in the milling industry because of the low operating cost, simple use for producers, and wide distribution in the agricultural sector.

Current state-of-the-art methodology relies mostly on LCMS, HPLC-UV and HPLC-FLD for the determination of several toxin concentrations. Especially, LC-MS methods are highly sensitive and have been comprehensively validated to provide documented accuracy and precision for regulatory purposes. Analyses require advanced laboratory facilities and highly qualified personnel, thus making these methods difficult and costly to use for food-processing companies. In contrast, spectroscopy-based rapid screening methods provide suitable and easy-to-use tools without the need for extensive laboratory facilities and highly qualified personnel (Kos et al., 2016). Accordingly, during the last decades, vibrational and optical spectroscopy-based techniques for the characterization and quantitation of fungal metabolites and toxins have been introduced. In particular, Fourier-transform-infrared (FTIR) spectroscopy has benefitted quality-control laboratories enabling rapid cost-effective results for DON determination with respect to numerous substrates, thus proving to be a robust and reliable technique (Dvořáček et al., 2012; Hossain and Goto, 2014; Ruan et al., 2002). Moreover, optical spectroscopy is currently emerging as a modern and green analytical technique for food analyses, due to the non-destructive nature of measurements (Ivanova and Spiteller, 2014; Mignani et al., 2016).
The selection of the desired technology for implementation in the food industry will depend on the purpose of the detection method used, the matrix of the specific sample, the required limit of detection (LOD), the cost, the equipment available, and operator training. The aim of this study was therefore to comparatively assess the usefulness of three analytical methods, differing in complexity and sensitivity for quantitation of the levels of DON - the predominant mycotoxin in the wheat-cropping area of Argentina - in samples of wheat and its by-products destined for commercial products.

\section{Materials and methods}

\section{Chemicals and materials}

Acetonitrile and methanol were purchased from J.T. Baker (Phillipsburg, NJ, USA) and ultrapure water produced by a Millipore Milli-Q system (Millipore, Bedford, MA, USA). A stock solution of DON (>99\% pure) at $1 \mathrm{mg} / \mathrm{ml}$ in acetonitrile was purchased from Sigma-Aldrich (St. Louis, MO, USA).

\section{Sample preparation}

We selected 20 representative samples from different regions of the Argentine pampas and at differing stages in commercial wheat-grain production, which specimens were received and stored in the mills until use. Due to the heterogeneous distribution of mycotoxins in wheat grains, the samples were treated equally for all determinations. Appropriate sampling procedures were used to obtain reliable results. Each sample (processed or whole) was ground and divided into four quadrants. After homogenisation, two opposite quadrants were remixed and redivided into four quadrants. The procedure was repeated until the amount of sample needed to perform all extractions was obtained. Three subsamples (about $100 \mathrm{~g}$ ) were obtained from each sample and stored in the freezer until the time of DON analysis.

\section{Deoxynivalenol extraction from wheat samples}

DON was extracted and purified by Mycosep 225 cleanup columns (Romer Labs Inc., Union, MO, USA). For this purpose, $25 \mathrm{~g}$ of milled wheat was added to $100 \mathrm{ml}$ of a solvent mixture consisting of acetonitrile:sterile water $(84: 16, \mathrm{v} / \mathrm{v})$ and blended for $3 \mathrm{~min}$ before filtration of the supernatant. Then, $8 \mathrm{ml}$ of the filtered supernatant was transferred to a glass tube and passed through the clean-up column; $4 \mathrm{ml}$ was recovered and evaporated to dryness. 


\section{Deoxynivalenol quantitation by high-performance liquid chromatography}

For the HPLC analysis, the extracts from the clean-up columns were reconstituted in $500 \mu \mathrm{l}$ of $12 \%(\mathrm{v} / \mathrm{v})$ aqueous methanol solution used in the mobile phase. From each extract, $50 \mu \mathrm{l}$ was injected onto a liquid-chromatography system (Waters 717plus Autosampler ${ }^{\mathrm{mw}}$; Waters Corporation, Milford, MA, USA) containing a C18 reverse-phase column ( $150 \times 4.6 \mathrm{~mm}, 5 \mu \mathrm{m}$ particle size; Waters Corporation) with an ultraviolet diode-array detector set at $220 \mathrm{~nm}$. The DON levels were detected as described by Cooney et al. (2001). The stock solution of DON $(1 \mathrm{mg} / \mathrm{ml})$ was used for preparing the working standard solutions (ranging from 0.5 to $5 \mu \mathrm{g} / \mathrm{ml}$ of DON) after evaporation of the acetonitrile from the stock solution under nitrogen and dissolution of the residue in an appropriate volume of mobile phase. Injections were made in triplicate and quantitation performed by means of Empower software (Chromatography Data System; Waters Corporation).

\section{Analysis of deoxynivalenol by lateral-flow devices}

The Reveal Q+ kit for DON (Neogen Corporation, Lansing, MI, USA) was provided by a local milling industry. The extraction of DON from the wheat samples was carried out as described by Van der Fels-Klerx and De Rijk (2014). Briefly, $10 \mathrm{~g}$ of each sample was ground, weighed, and suspended in $100 \mathrm{ml}$ of distilled water. The mixture was vigorously shaken for $30 \mathrm{~min}$, filtered, and then directly tested with the kit as indicated by the manufacturer. Of the sample diluent provided by the manufacturer, $1000 \mu \mathrm{l}$ was added to the antibody-coated red dilution microwell of the Reveal-Q+ test plate before addition of $100 \mu \mathrm{l}$ of the sample extract. The contents of the well were mixed vigorously by pipetting five times, and $100 \mu \mathrm{l}$ were then transferred to a sample cup. A Reveal-Q+ strip was placed into the sample cup and after 3 min the strip was placed in the AccuScan Pro Reader $^{\text {ntw }}$ (Neogen Corporation). The range of DON quantitation specified for this technique according to the kit's instructions is between 0.3 and $6 \mu \mathrm{g} / \mathrm{g}$ of sample.

\section{FTIR spectroscopy: spectral acquisition and data analysis}

FTIR-spectroscopy measurements were made through the application of dried-film technology (Naumann, 2000). For this purpose, the purified extracts obtained from the wheat samples by means of the Mycosep columns were reconstituted in $200 \mu \mathrm{l}$ of distilled water and an aliquot of $80 \mu \mathrm{l}$ transferred to $\mathrm{ZnSe}$ optical plates (13 mm diameter, Korth Kristalle GMBH, Altenholz, Germany). Samples were further dried at $40^{\circ} \mathrm{C}$ for $40 \mathrm{~min}$ to produce transparent films (Bosch et al., 2006; Naumann, 2000).
FTIR spectra were obtained between 4,000 and $650 / \mathrm{cm}$ with 64 scans at $6 / \mathrm{cm}$ of spectral resolution in a Spectrum One $^{\mathrm{sm}}$ FTIR spectrometer (Perkin-Elmer Instruments, Waltham, MA, USA). Before data analysis, raw spectra were subjected to a data-preprocessing procedure consisting in a baseline correction (by the rubber-band-correction method involving a 40-point baseline and 10 interactions) and calculation of second derivatives (through the use of the Savitzky-Golay 9-point smoothing algorithm) in order to enhance the spectral resolution of overlapping bands. A semi-quantitative estimation of DON was performed with the preprocessed spectra by calculating the so-called $\beta$-ratio, which parameter was obtained from the area of the band at $1,018 / \mathrm{cm}(1,140-980 / \mathrm{cm})$ - assigned to the of the C-O-C vibrational mode in DON molecules - divided by the area of the band at $2,920 / \mathrm{cm}(2,946-2,983 / \mathrm{cm})-$ the result of C-H stretching in methylene $\left(-\mathrm{CH}_{2}-\right)$ groups, those being used as a reference for the total amount of sample (Naumann, $2000)$ - i.e. $\beta=A_{1,018} / A_{2,920}$. The frequencies, the number of peaks to be fitted in the region, and the half-width of each peak used to start a least-squares iterative curve-fitting procedure were those obtained from the second derivative of the original spectra (Fabian and Schultz, 2000; Ferrer et al., 2008). The areas of the bands were calculated by integration of the corresponding fitted bands. For a best fit, a straight baseline passing through the ordinates at 1,140 and $980 / \mathrm{cm}$ was adjusted. The curve fitting was performed by a stepwise iterative adjustment towards a minimum rootmean-square error of the different parameters determining the shape and position of the absorption peaks. This step was carried out by assuming an initial mixed LorentzianGaussian line-shape function, with a full-width band at half-height of 20-50/cm and a maximum resolution factor. Data preprocessing, curve fitting, and peak-area calculation were carried out by means of the OPUS software (version 4.0 and 7.0, Bruker Optics GmbH, Ettlingen, Germany). The semi-quantitative determination of DON was obtained from the average of three $\beta$-values calculated from the spectra acquired from independent extractions.

\section{Validation of analytical method}

Validation of the analytical methods was based on the criteria of linearity, specificity, reproducibility, LOD, limit of quantification (LOQ) and recovery. The linearity of each method was confirmed using the calibration curve. The calibration curves were constructed with different DON concentrations from 0.5 to $5 \mu \mathrm{g} / \mathrm{ml}$. Linearity was shown with the correlation coefficient $\left(\mathrm{R}^{2}\right)$ through linear regression analysis. The selectivity of the method was determined through the comparative analysis of DON free wheat samples and spiked wheat samples at 0.1, 2 and $10 \mu \mathrm{g} / \mathrm{g}$ of DON. The sensitivity of the methods was assessed using the method LOD and LOQ. The LOD was defined as the minimum concentration of an analyte in the sample inducing multiple reaction monitoring mode with 
a signal-to-noise $(\mathrm{S} / \mathrm{N})$ ratio of at least 3 while an $\mathrm{S} / \mathrm{N}$ ratio of 10 was considered as the method LOQ. The recovery process was set by contaminating DON free samples of wheat with above mentioned DON concentrations on the same HPLC conditions.

\section{Statistical analysis}

The Pearson correlation coefficient was used to evaluate the strength of the relationships among the DON determinations by HPLC, Reveal Q+, and FTIR spectroscopy.

\section{Results and discussion}

In order to assist the producer in choosing the most appropriate tool according to the purpose of the analysis, we put the different techniques available within the food industry for determining DON levels in wheat and wheat by-products side by side for comparison. The performances of both the competitive immunochromatographic assay and the technique of absorbance-and-transmission FTIR spectroscopy for toxin detection were analysed and compared with HPLC, the latter being a widely accepted method of standard reference, in order to obtain the values of the DON content in all the reference samples of wheat tested (Table 1).

\section{Validation of analytical method}

According to the regression analysis, calibration curves for DON were linear from all analysed range for the three investigated methods. Mycotoxin recoveries varied considerably, depending on the DON concentration; the variation generally decreased with increasing toxin concentration. The reproducibility, LOD, LOQ and mean recoveries are presented in Table 2. These results suggest that the chosen analytical methods exhibited good accuracy and precision for the detection of DON in these wheat samples.

\section{HPLC quantitation}

The DON content of the samples was quantified by HPLC with ultraviolet detection. This technique is recognised as the most widely used method of DON screening, due to its high degree of accuracy, specificity, and reproducibility. HPLC was also adopted by the International Association of Analytical Communities and the European Committee for Standardization for the determination of DON (Meenely

Table 1. Concentration of deoxynivalenol (DON) estimated by different analytical techniques on wheat samples. ${ }^{1}$

\begin{tabular}{|c|c|c|c|c|}
\hline \multirow[t]{2}{*}{ Sample ID } & \multirow[t]{2}{*}{ Samples $^{2}$} & \multicolumn{2}{|c|}{ DON ( $\mu \mathrm{g} / \mathrm{g} \pm$ standard deviation) } & \multirow[t]{2}{*}{ FTIR ( $\beta$-value) } \\
\hline & & HPLC & Reveal Q+ & \\
\hline 1 & durum wheat & ND & ND & $0.18 \pm 0.03$ \\
\hline 2 & durum wheat & ND & $0.10 \pm 0.00$ & $0.25 \pm 0.03$ \\
\hline 3 & soft wheat & ND & $0.30 \pm 0.05$ & $0.56 \pm 0.05$ \\
\hline 4 & soft wheat & ND & $0.40 \pm 0.12$ & $0.57 \pm 0.01$ \\
\hline 5 & flour 000 & $0.16 \pm 0.01$ & $0.15 \pm 0.05$ & $0.27 \pm 0.05$ \\
\hline 6 & flour 000 & $0.17 \pm 0.02$ & $0.15 \pm 0.04$ & $0.39 \pm 0.01$ \\
\hline 7 & flour 000 & $0.17 \pm 0.05$ & $0.10 \pm 0.00$ & $0.33 \pm 0.01$ \\
\hline 8 & wheat from the initial cleaning & $0.28 \pm 0.04$ & $0.30 \pm 0.01$ & $0.54 \pm 0.01$ \\
\hline 9 & flour 000 & $0.29 \pm 0.03$ & $0.25 \pm 0.05$ & $0.43 \pm 0.03$ \\
\hline 10 & soft wheat & $0.31 \pm 0.03$ & $0.10 \pm 0.00$ & $0.64 \pm 0.00$ \\
\hline 11 & flour 000 & $0.38 \pm 0.03$ & $0.25 \pm 0.05$ & $0.61 \pm 0.01$ \\
\hline 12 & soft wheat & $0.41 \pm 0.40$ & $0.25 \pm 0.02$ & $0.61 \pm 0.01$ \\
\hline 13 & wheat with impurities & $0.45 \pm 0.05$ & $0.80 \pm 0.07$ & $0.67 \pm 0.02$ \\
\hline 14 & bran & $0.56 \pm 0.18$ & $1.40 \pm 0.36$ & $0.73 \pm 0.02$ \\
\hline 15 & fine by-products of wheat milling & $1.00 \pm 0.20$ & $1.55 \pm 0.20$ & $0.93 \pm 0.05$ \\
\hline 16 & mixture of soft and durum wheat & $1.09 \pm 0.20$ & $1.45 \pm 0.22$ & $0.99 \pm 0.00$ \\
\hline 17 & fine product of wheat milling & $1.30 \pm 0.04$ & $0.75 \pm 0.23$ & $1.52 \pm 0.13$ \\
\hline 18 & soft wheat & $2.30 \pm 0.47$ & $2.90 \pm 0.87$ & $2.58 \pm 0.12$ \\
\hline 19 & durum wheat & $2.50 \pm 0.02$ & $2.54 \pm 0.31$ & $2.31 \pm 0.12$ \\
\hline 20 & fraction from a cleaning of the destoner machine & $14.20 \pm 3.72$ & $>7$ & $12.4 \pm 0.50$ \\
\hline
\end{tabular}


Table 2. Validation parameters for determining deoxynivalenol concentrations in $\mathbf{2 0}$ contaminated wheat samples destined for the manufacture of commercial products using different analytical techniques. ${ }^{1}$

\begin{tabular}{llll}
$\begin{array}{l}\text { Validation } \\
\text { parameters }\end{array}$ & HPLC & Reveal Q+ & FTIR \\
& & & \\
$\mathrm{RSD}_{\mathrm{r}}(\%)^{2}$ & 1.57 & 3.33 & 4.00 \\
Detection limit & $0.12(\mu \mathrm{g} / \mathrm{g})$ & - & $0.12(\beta$-value $)$ \\
Quantification limit & $0.30(\mu \mathrm{g} / \mathrm{g})$ & $0.3(\mu \mathrm{g} / \mathrm{g})$ & $0.15(\beta$-value $)$ \\
Recovery $(\%)^{3}$ & $87-95$ & $76-88$ & $82-93$ \\
$r^{24}$ & 0.981 & $*$ & $*$ \\
\hline
\end{tabular}

${ }^{1}$ HPLC = high-performance liquid chromatography; Reveal Q+ = a lateralflow device; FTIR = Fourier-transform infrared spectroscopy; ND = not detected.

${ }^{2}$ Mean relative standard deviation for repeatability.

${ }^{3}$ The value represent the mean toxin recoveries within all assayed concentrations ( $n=4$ replicates)

${ }^{4}$ Regression coefficient of the method; * no standard curve was developed.

et al., 2011). The DON levels in the 20 wheat and wheat by-product samples varied from 'not detected' (indicated in Table 1 for values below the LOD) to $14.2 \mu \mathrm{g} / \mathrm{g}$, with a mean value of $1.3 \mu \mathrm{g} / \mathrm{g}$. This concentration range covered the majority of the DON levels found in routine analyses performed in wheat mills in order to evaluate the quality of both the incoming raw materials and the potential wheat by-products (Table 1 ).

The analysis of these samples was performed with the objective of determining specificity - i.e. discriminating between the desired and the unwanted components present. In addition, the purity of the peaks was determined by performing a spectral-homogeneity test with Empower PDA Software (Waters Corporation). The samples analysed indicated a degree of purity below the impurity threshold determined by the program, confirming the spectral homogeneity. The reproducibility of the method enabled a determination of results over a short time interval under identical conditions (i.e. the interassay precision) that gave a relative standard deviation of $1.57 \%$. The ability of a testing method to provide results directly proportional to the analyte concentration within a given range (linearity) has been reported as the variance of the slope of the regression line (standard error $0.2296, \mathrm{R}^{2}=0.9871$ ). The LOD and LOQ of this method were $0.12 \mu \mathrm{g} / \mathrm{g}$ and $0.3 \mu \mathrm{g} / \mathrm{g}$, respectively.

The Mycosep clean-up columns applied in this study as a purification procedure also proved to be suitable for DON determination. Previous studies have utilised this preparative method for the simultaneous detection of DON and other trichothecenes in cereal grains (Berthiller et al., 2005a,b; Böhm et al., 2008; Klinglmayr et al., 2009; Neuhof et al., 2009); this technique representing one of the most widely used approaches for the extraction of DON.

While for the separation and detection of DON from wheat and by-products, gas chromatography (Schothorst and Jekel, 2001; Vega and Castillo, 2006) and liquid chromatography (Makun et al., 2011; Sebaei et al., 2012; Trucksess et al., 2010) were widely used, more recently, the favoured method of detection DON from several foodstuff matrixes has shifted to tandem mass spectrometry (Boutigny et al., 2012; Goertz et al., 2010; Ji et al., 2014; Liu et al., 2016; Tanaka et al., 2000; Tralamazza et al., 2016) which has greater flexibility in simultaneously detecting and identifying various groups of these metabolites, as well as reaching lower detection limits than those obtained in this study. However, in the range of the assayed concentrations, the detection limits obtained by the three tested methods proved useful, reliable and suitable according to the current legislation for its commercialization.

\section{Analysis by lateral-flow devices}

Table 1 delineates the DON concentrations obtained by the lateral-flow immunochromatographic technique for the 20 samples of wheat and the associated by-products. These results properly demonstrated the usefulness of the Reveal-Q+ device for the milling industry in view of the method's advantages in expediting commodities and products through marketing channels, thus saving time and costs and enabling the employment of staff with little technical training. Nevertheless, as indicated by the HPLC results, these advantages are gained at the expense of lower accuracy and precision. In this regard, we wish to stress that although the limit of quantitation given by the manufacturers of the Reveal Q+ is stated to be $0.3 \mu \mathrm{g} / \mathrm{g}$, our results indicated an excellent correlation ( 0.92$)$ between the HPLC and the Reveal Q+ method at a very high level of significance $(P<0.0001)$ when DON concentrations were within the range between 0.1 and $0.3 \mu \mathrm{g} / \mathrm{g}$.

The results with the Reveal Q+ kit for DON in the present study were in agreement with those obtained by numerous authors, who also observed a certain dispersion in the values measured with the commercial kits and those obtained by the analytical method used as the reference technique. Matthews and Pratt (2007) and Tangni et al. (2010) found little difference in a comparison to DON concentrations obtained by the commercial kit RosaFast DON ${ }^{\mathrm{TM}}$ (Charm Sciences Inc., Lawrence, MA, USA) with those provided by liquid-chromatography-mass-spectrometry. In contrast, Maragos and Plattner (2002) observed that the kit RidaQuick DON ${ }^{\mathrm{sm}}$ (R-Biopharm AG, Darmstadt Germany) overestimated the DON concentrations because of the extensive antibody cross-reactions. In addition, Van der Fels-Klerx and De Rijk (2014), in a recent evaluation of the 
performance of these lateral-flow-immunoassay test kits for DON quantitation in wheat, observed that the recovery of the RidaQuick kit was the lowest.

\section{FTIR spectroscopy detection and semi-quantitative analysis}

Infrared spectroscopy has found widespread use in the analysis of chemical compounds in various materials related to the food industry. For example, different approaches employing mid-infrared spectroscopy have been previously applied to DON detection and quantitation in grains, with most of those techniques being based on attenuated total reflectance and/or drift reflection (Abramovic et al., 2007; Kos et al., 2002, 2003). In the work reported here, we applied dried-film technology (Naumann, 2000) to absorbancetransmission spectroscopy in order to semi-quantitate the DON levels in wheat samples. Figure 1A shows an overlay of the average FTIR spectra acquired from three different wheat samples (IDs: 11, 14, and 17) containing dissimilar amounts of DON along with the spectrum of one of the DON standards used for the calibration curve. These IR spectra provide information on the chemical composition of the wheat extracts recovered after the clearance step. The band positions and shapes and the relative peak intensities of the average spectra obtained from each sample were analysed in the following spectral regions: $\mathrm{W}_{1}, 3,000-2,800 / \mathrm{cm}$, known as the lipid region and containing the bands attributed to the symmetric and antisymmetric stretching modes of the $\mathrm{C}-\mathrm{H}$ bond in the methyl $\left(\mathrm{CH}_{3}-\right)$ and methylene $\left(-\mathrm{CH}_{2}-\right)$ groups; $\mathrm{W}_{2}, 1,700$ $1,580 / \mathrm{cm}$, containing the amide-I and amide-II bands, with $\mathrm{C}=\mathrm{O}$ stretching vibrations belonging to esters and amides along with the $\mathrm{N}-\mathrm{H}$ bending and some contribution of the C-N stretching vibrations; $\mathrm{W}_{3}, 1,450-1,350 / \mathrm{cm}$ (the mix region); and finally $\mathrm{W}_{4}, 1,200-900 / \mathrm{cm}$, corresponding to a broad band that comprises the peaks mainly assigned to the stretching modes of carbohydrate rings and side groups (e.g. C-O-C, C-OH, C-H; cf. Bosch et al., 2010; Helm and Naumann, 1995; Kacurakova et al., 2000; Naumann, 2000).

The spectra of both the DON standards and the wheat samples displayed a band in the region 1,100-980 that could be deconvoluted into three peaks $(1,106,1,048,1,018$ / $\mathrm{cm}$ ) with the one at $1,018 / \mathrm{cm}$ proving to be sensitive to the DON concentration (Figure 1B) for all the wheat samples analysed. We wish to emphasise that previous studies performed on DON detection and quantitation by infrared spectroscopy in wheat samples reported the ester band at $1,750-1,700 / \mathrm{cm}$ to be sensitive to the DON concentration (Abramovic et al., 2007). Nevertheless, when an enhanced-resolution technique, such as the secondderivative analysis, was applied to the spectra obtained from the samples studied here, we found that the band at 1,750$1,700 / \mathrm{cm}$ was not sensitive to DON concentration. In the majority of the samples, this broad band was the result of two or three $\mathrm{C}=\mathrm{O}$ stretching contributions - depending on the sample - at 1,735-1,740, 1,729, 1,714-1,712/cm, owing to the $\mathrm{COOR}, \mathrm{COOH}$, and $\mathrm{COO}$ - moieties, respectively (Naumann, 2000). Thus, although both the peak area and the peak height were sensitive to DON concentrations in the spectra from the samples of the DON calibration curve, those parameters proved to be not so sensitive in the wheat samples that we analysed. Nevertheless, we found that the spectra of both the DON standards and the wheat samples displayed a band in the region $1,100-980 / \mathrm{cm}$ that, when deconvoluted, revealed a peak at $1,018 / \mathrm{cm}$ that proved to be sensitive for DON detection in all wheat samples with concentrations higher than $0.15 \mu \mathrm{g} / \mathrm{g}$. Therefore, on the basis of this consideration, the semi-quantitative determination of DON was achieved by calculating the $\beta$-ratio with these data: i.e. $A_{1,018} / A_{2,920}$ (Table 1).

The LOD of DON by FTIR measurements was determined by the minimum concentration at which the peak at $1,018 / \mathrm{cm}$ could be detected in the spectrum, which level corresponded to $0.12 \beta$-value of wheat, while the limit of semi-quantification was determined by the minimum area of the peak at $1,018 / \mathrm{cm}$ that could be calculated by the software (i.e. an area $\geq 0.025$ ), corresponding to a DON content of $>0.15 \beta$-value. Other authors using (FT) IR spectroscopy employ the technique with attenuated total reflectance or diffuse reflectance on raw samples (they did not extract) and classify the spectra in ranges or classes according to the values obtained by goldstandard methodologies to estimate DON concentrations in agricultural commodities (De Girolamo et al., 2014; Hossain and Goto, 2014; Kos et al., 2016). In this paper, we implement the technique of dry film technology on extracts and apply a semi quantitative method which allowed to achieve greater precision and better detection limits. The reproducibility of the semi-quantification performed by FTIR spectroscopy depends on the DON concentration in the sample: the relative standard deviation of the $\beta$-value obtained for the three independent replicates varied from 3 to $8 \%$ (Table 1 ).

The dried-film-FTIR-spectroscopy-based approach offers multiple advantages for DON detection and quantification. The $\beta$-values generated by the technique correlated well with the data obtained by HPLC. Moreover, the detection method does not involve the addition of solvents or toxic substances and is rapid since the extracts obtained from the clean-up columns are simply reconstituted in water, placed onto the ZnSe window, dried, and measured. No additional sample preparation is required, thus maintaining a low cost in operation.

In brief, the dried-film-FTIR-spectroscopy method was successfully used for determining and quantifying DON from wheat and by-products obtaining very good detection limits values comparable with those obtained by other 

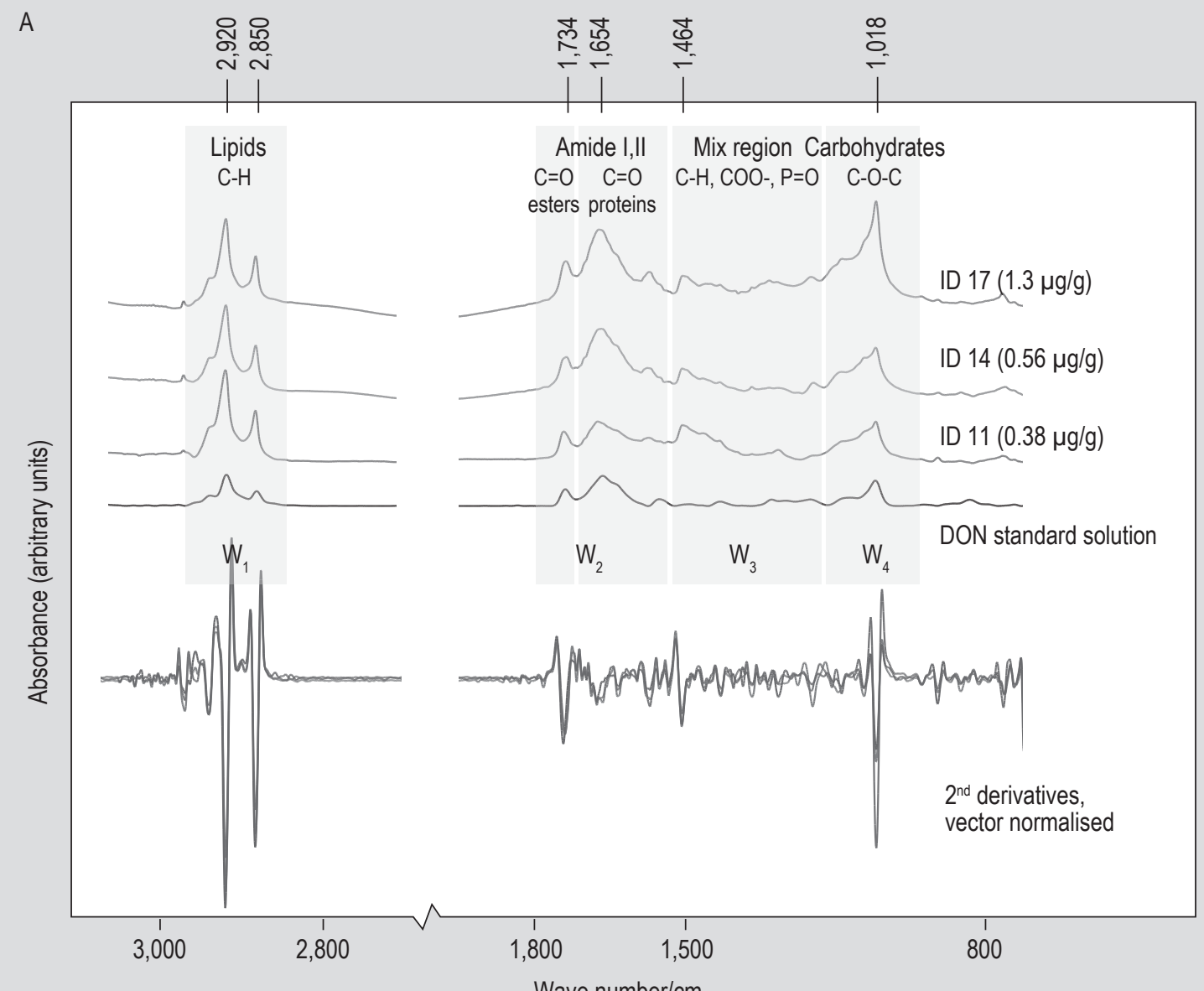

B

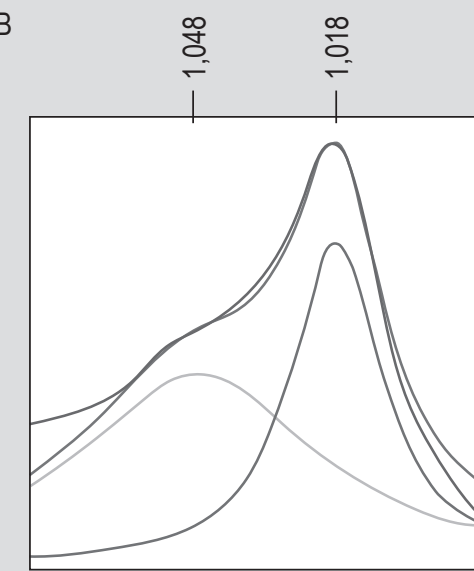

ID $17: 1.3 \mu \mathrm{g} / \mathrm{g}$

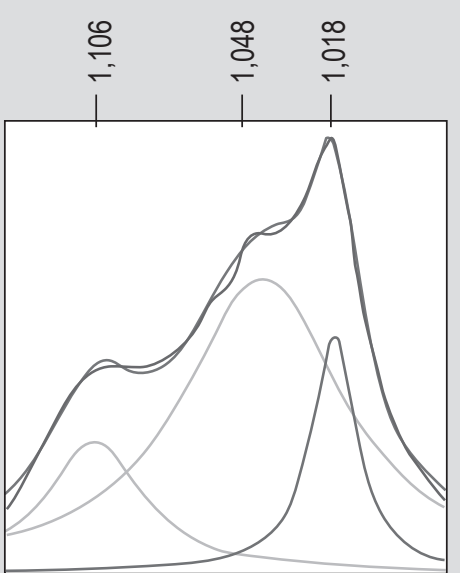

ID $14: 0.56 \mu \mathrm{g} / \mathrm{g}$ $\beta=0.73$

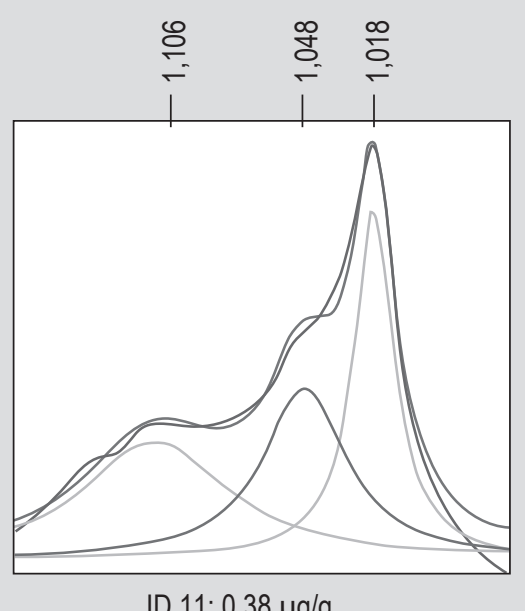

ID 11: $0.38 \mu \mathrm{g} / \mathrm{g}$ $\beta=0.61$

Figure 1. (A) Overlay of the average Fourier-transform-infrared (FTIR)-absorbance spectra from replicates of three different wheat samples (IDs 11, 14, and 17) containing increasing deoxynivalenol (DON) levels $(0.38,0.56$, and $1.3 \mu \mathrm{g} / \mathrm{g}$ of DON as measured by HPLC). The spectral windows and band assignments are indicated. (B) Semiquantification of DON by curve-fitting and estimation of the $\beta$ coefficient, calculated as the ratio of the areas under the peaks from Figure $1 A$ at $1,018 / \mathrm{cm}$ (resulting from DON absorption) and $2,920 / \mathrm{cm}$ (considered as representative of the total amount of sample).

optical spectroscopy techniques, which are currently emerging as modern and 'green' analytical techniques for food analyses, such as Raman spectroscopy which also demonstrated good potentials for non-destructive and rapid screening of DON-contaminated wheat samples (Liu et al., 2009; Mignani et al., 2016). These methods, however, are 
hindered by the intense and broad spectral bands attributed to naturally occurring moisture. Raman spectroscopy could be an alternative to IR and NIR due to its insensitivity to water and fewer overlapping bands. Since non-polar groups give intense absorbance's in Raman spectra and polar groups cause high-absorption peaks in FTIR spectroscopy, the use of both spectroscopic techniques in a complementary fashion can provide important and complete information (Ma and Phillips, 2002). An important difference to remark between these techniques is that the $\mathrm{O}-\mathrm{H}$ stretching vibration is very strong in IR, but very weak in Raman spectra. For this reason, water is practically 'invisible' in Raman spectroscopy, while it dominates the IR spectrum, if present. An another great difference is that the FTIR equipment is relatively inexpensive compared to Raman equipment (Ferrer et al., 2008).

\section{Correlation obtained among HPLC, FTIR, and Reveal-Q+ methodologies}

Significant Pearson correlation coefficients were obtained among the results obtained by the three assay methods.
A strongly positive correlation at a high significance level was observed between HLPC and the semi-quantitative $\beta$-value calculated by the FTIR method, unlike the low degree of correlation seen with the data obtained by Reveal Q+ (Table 3, analysis A). We therefore decided to repeat the correlation analysis considering only the whole-wheat (i.e. durum or soft) and wheat-flour samples and rejecting, at once, samples from the wheat with impurities, the bran, the fine products and by-products of the wheat milling, and the fraction from the cleaning of the destoner machine (Table 1 ; numbers $13,14,15,17$, and 20 , respectively) since the impurities present in the products or by-products arising during the processing of the wheat flour probably have other compositional characteristics that do not allow a satisfactory DON detection and also influence the subsequent DON extraction and quantification (Table 3 , analysis B).

\section{Conclusions}

Table 4 summarizes the main features of each of the methods used, and states the advantages and disadvantages in the implementation of those methods in the milling industry.

Table 3. Pearson's correlation coefficient among DON concentrations obtained by HPLC, Reveal Q+, and FTIR spectroscopy.

\begin{tabular}{|c|c|c|c|c|c|}
\hline \multirow[t]{2}{*}{ Analysis $^{1}$} & \multirow[t]{2}{*}{ Methods } & \multicolumn{2}{|l|}{ FTIR } & \multicolumn{2}{|l|}{ Reveal Q+ } \\
\hline & & Correlation value & $P$-value & Correlation value & $P$-value \\
\hline \multirow[t]{2}{*}{ A } & HPLC & 0.94 & $<0.0001$ & 0.45 & 0.0003 \\
\hline & FTIR & & & 0.46 & 0.0002 \\
\hline \multirow[t]{2}{*}{ B } & HPLC & 0.95 & $<0.0001$ & 0.70 & $<0.0001$ \\
\hline & FTIR & & & 0.75 & $<0.0001$ \\
\hline
\end{tabular}

${ }^{1}$ The data pool was conformed by a value for $\mathrm{n}$ in $\mathrm{A}$ of 60 and in $\mathrm{B}$ of 45 .

Table 4. Comparison of the three analytical methods for detection of deoxynivalenol (DON).

\begin{tabular}{|c|c|c|c|}
\hline $\begin{array}{l}\text { Analytical } \\
\text { methods }^{1}\end{array}$ & Main characteristics & Advantages & Disadvantages \\
\hline HPLC & $\begin{array}{l}\text { - gold standard method } \\
\text { - suitable for DON quantitation in a wide } \\
\text { range of concentrations, even low levels }\end{array}$ & $\begin{array}{l}\text { - excellent sensitivity } \\
\text { - good selectivity } \\
\text { - good repeatability and reproducibility }\end{array}$ & $\begin{array}{l}\text { - expensive equipment } \\
\text { - expensive accessories are required } \\
\text { (e.g. carriers, columns) } \\
\text { - samples need a clean-up step }\end{array}$ \\
\hline Reveal Q+ & $\begin{array}{l}\text { - suitable for rapid screening in the milling } \\
\text { industry } \\
\text { - commercial test kit available }\end{array}$ & $\begin{array}{l}\text { - rapid and simple } \\
\text { - single step and low cost } \\
\text { - no specific training required } \\
\text { - can be used in situ }\end{array}$ & $\begin{array}{l}\text { - low sensitivity (cross-reactivity) } \\
\text { - possible false positive }\end{array}$ \\
\hline FTIR & $\begin{array}{l}\text { - no accessories are required after DON } \\
\text { extraction }\end{array}$ & $\begin{array}{l}\text { - good sensitivity } \\
\text { - rapid detection } \\
\text { - expertise is not required }\end{array}$ & $\begin{array}{l}\text { - relatively expensive instrument } \\
\text { - samples need a previous clean-up step } \\
\text { - semi-quantitative method }\end{array}$ \\
\hline
\end{tabular}

${ }^{1}$ HPLC = high-performance liquid chromatography; Reveal Q+ = a lateral-flow device; FTIR = Fourier-transform infrared spectroscopy. 
The results obtained in this work have indicated that both methods of assay - Reveal Q+ and FTIR-spectroscopy - are effective for DON detection from samples of wheat and the commercial by-products according to the sensitivity required by the milling sector.

The FTIR-spectroscopic dried-film technology, used here for the first time for DON detection, constitutes an alternative approach for screening a large number of samples of wheat and the commercial by-products in order to determine whether the DON concentrations assayed are in conformity with the international regulations.

In addition, this study compares the complexity of the two methods for extracting DON depending on the matrix and also demonstrates that the Reveal-Q+ approach could be more precise for the quantification of DON in samples of whole wheat (durum or soft) and of wheat flour. Therefore, the protocol for DON extraction from samples originating during the elaboration of wheat flour should be accordingly reformulated.

\section{Acknowledgements}

We thank Consejo Nacional de Investigaciones Científicas y Tecnológicas (Grants PIP 0925), and FONCYT, PICT: 2011-0851 for financial support and are grateful for the technical assistance of Esteban Manuel González. A. Bosch is member of CIC PBA. Dr. Donald F. Haggerty, a retired academic career investigator and native English speaker, edited the final version of the manuscript.

\section{References}

Abramovic, B., Jajic, I., Abramovic, B., Cosic, J. and Juric, V., 2007. Detection of deoxynivalenol in wheat by Fourier transform infrared spectroscopy. Acta Chimica Slovenica 54: 859-867.

Alldrick, A.J., Van Egmond, H.P. and Solfrizzo, M., 2009. Commercial use of rapid mycotoxin test kits: significance and potential harmonisation issues. World Mycotoxin Journal 2: 215-220.

Berthiller, F., Dall'Asta, C., Schuhmacher, R., Lemmens, M., Adam, G. and Krska, R., 2005a. Masked mycotoxins: determination of a deoxynivalenol glucoside in artificially and naturally contaminated wheat by liquid chromatographyetandem mass spectrometry. Journal of Agricultural and Food Chemistry 53: 3421-3425.

Berthiller, F., Schuhmacher, R., Buttinger, G. and Krska, R., 2005b. Rapid simultaneous determination of major type Aand $\mathrm{B}$-trichothecenes as well as zearalenone in maize by high performance liquid chromatographyetandem mass spectrometry. Journal of Chromatography A 1062: 209-216.

Böhm, C., Cichna-Markl, M., Brenn-Struckhofova, Z. and RazzaziFazeli, E., 2008. Development of a selective sample clean-up method based on immunoultrafiltration for the determination of deoxynivalenol in maize. Journal of Chromatography A 1202: 111-117.
Bosch, A., Golowczyc, M., Abraham, A.G., Garrote, G., De Antoni, G.L. and Yantorno, O., 2006. Rapid discrimination of lactobacilli isolated from kefir grains by FT-IR spectroscopy. International Journal of Food Microbiology 111: 280-287.

Bosch, A., Prieto, C., Serra, D.O., Martina, P., Stämmler, M., Naumann, D., Schmitt, J. and Yantorno, O., 2010. Type-IV pili spectroscopic markers: applications in the quantification of piliation levels in Moraxella bovis cells by a FT-IR ANN-based model. Journal of Biophotonics 3: 522-533.

Boutigny, A., Beukes, I., Small, I., Zuehlke, S., Spiteller, M., Van Rensburg, B., Flett, B. and Viljoen, A., 2012. Quantitative detection of Fusarium pathogens and their mycotoxins in South African maize. Plant Pathology 61: 522-531.

Cooney, J.M., Lauren, D.R. and Di Menna, M.E., 2001. Impact of competitive fungi on trichothecene production by Fusarium graminearum. Journal of Agricultural and Food Chemistry 49: 522-526.

De Girolamo, A., Cervellieri, S., Visconti, A. and Pascale, M., 2014. Rapid analysis of deoxynivalenol in durum wheat by FT-NIR Spectroscopy. Toxins 6: 3129-3143.

Dvořáček, V., Prohasková, A., Chrpová, J. and Štočková, L., 2012. Near infrared spectroscopy for deoxynivalenol content estimation in intact wheat grain. Plant, Soil and Environment 58: 196-203.

European Commission (EC), 2007. Commission Regulation (EC) No 1126/2007 of 28 September 2007 amending Regulation (EC) No $1881 / 2006$ setting maximum levels for certain contaminants in foodstuffs as regards Fusarium toxins in maize and maize products. Official Journal of the European Union L 255: 14-17.

Fabian, H. and Schultz, C.P., 2000. Fourier transform infrared spectroscopy in peptide and protein analysis. In: Meyer, R.A. (ed.) Encyclopedia of analytical chemistry. John Wiley and Sons, Chichester, UK, pp. 57-79.

Ferrer, E.G. Bosch, A., Yantorno, O. and Baran, E.J., 2008. A spectroscopy approach for the study of the interactions of bioactive vanadium species with bovine serum albumin. Bioorganic and Medicinal Chemistry 16: 3878-3886.

Goertz, A., Zuehlke, S., Spiteller, M., Steiner, U., Dehne, H., Waalwijk, C., De Vries, I. and Oerke, E., 2010. Fusarium species and mycotoxin profiles on commercial maize hybrids in Germany. European Journal of Plant Pathology 128: 101-111.

Helm, D. and Naumann, D., 1995. Identification of some bacterial cell components by FT-IR spectroscopy. FEMS Microbiology Letters 126: $75-80$.

Hossain, M. and Goto, T., 2014. Near- and mid-infrared spectroscopy as efficient tools for detection of fungal and mycotoxin contamination in agricultural commodities. World Mycotoxin Journal 7: 507-515.

Ivanova, B. and Spiteller, M., 2014. Raman spectroscopic and mass spectrometric determination of aflatoxins. Food Analytical Methods 7: 242-256.

Ji, F., Xu, J., Liu, X., Yin, X. and Shi, J., 2014. Natural occurrence of deoxynivalenol and zearalenone in wheat from Jiangsu province, China. Food Chemistry 157: 393-397.

Kacurakova, M., Capek, P., Sasinkova, V., Wellner, N. and Ebringerova, A., 2000. FT-IR study of plant cell wall model compounds: pectic polysaccharides and hemicelluloses. Carbohydrate Polymers 43: 195-203. 
Klinglmayr, C., Nobauer, K., Razzazi-Fazeli, E. and Cichna-Markl, M., 2009. Determination of deoxynivalenol in organic and conventional food and feed by sol-gel immunoaffinity chromatography and HPLC-UV detection. Journal of Chromatography B 878: 187-193.

Koch, P., 2004. State of the art of trichothecenes analysis. Toxicological Letters 153: 109-112.

Kos, G., Lohninger, H. and Krska, R., 2002. Fourier transform midinfrared spectroscopy with attenuated total reflection (FT-IR/ATR) as a tool for the detection of Fusarium fungi on maize. Vibrational Spectroscopy 29: 115-119.

Kos, G., Lohninger, H. and Krska, R., 2003. Development of a method for the determination of Fusarium fungi on corn using mid-infrared spectroscopy with attenuated total reflection (FTIR-ATR) and chemometrics. Analytical Chemistry 75: 1211-1217.

Kos, G., Sieger, M., McMullin, D., Zahradnik, C., Sulyok, M., Öner, T., Mizaikoff, B. and Krska, R., 2016. A novel chemometric classification for FTIR spectra of mycotoxin-contaminated maize and peanuts at regulatory limits. Food Additives and Contaminants Part A 33; 1596-1607.

Liu, Y., Delwiche, S.R. and Dong, Y., 2009. Feasibility of FT-Raman spectroscopy for rapid screening for DON toxin in ground wheat and barley. Food Additives and Contaminants Part A 26: 1396-1401.

Liu, Y., Lu, Y., Wang, L., Chang, F. and Yang, L., 2016. Occurrence of deoxynivalenol in wheat, Hebei Province, China. Food Chemistry 197: 1271-1274.

Ma, C. and Phillips, D.L., 2002. FT-Raman spectroscopy and its applications in cereal science. Cereal Chemistry Journal 79: 171-175.

Makun, H.A., Dutton, M.F., Njobeh, P.B., Mwanza, M. and Kabiru, A.Y., 2011. Natural multi-occurrence of mycotoxins in rice from Niger State, Nigeria. Mycotoxin Research 27: 97-104.

Maragos, C.M. and Plattner, R.D., 2002. Rapid fluorescence polarization immunoassay for the mycotoxin deoxynivalenol in wheat. Journal of Agricultural and Food Chemistry 50: 1827-1832.

Matthews, N.J. and Pratt, J.M., 2007. Extension to HGCA project report no. 394: evaluation of test kits for deoxynivalenol (DON). Evaluation of Rosa DON P/N Test and Rosa DON (Quantitative) Test. Campden and Chorleywood Food Research Association, Gloucestershire, UK. Available at: http://tinyurl.com/hx4k6eu.

Meneely, J.P., Ricci, F., Van Egmond, H.P. and Elliott, C., 2011. Current methods of analysis for the determination of trichothecene mycotoxins in food. Trends in Analytical Chemistry 30: 192-203.

Mignani, A., Ciaccheri, L., Mencaglia, A., De Girolamo, A., Lippolis, V. and Pascale, M., 2016. Rapid screening of wheat bran contaminated by deoxynivalenol mycotoxin using Raman spectroscopy: a preliminary experiment. In: Proceedings of the $6^{\text {th }}$ European Workshop on Optical Fibre Sensors, the International Society for Optical Engineering, Bellingham, WA, USA.
Naumann, D., 2000. Infrared spectroscopy in microbiology. In: Meyers, R.A. (ed.) Encyclopedia of analytical chemistry: applications, theory, and instrumentation. John Wiley and Sons Ltd., Chichester, UK, pp. 102-131.

Neuhof, T., Ganzauer, N., Koch, M. and Nehls, I., 2009. A comparison of chromatographic methods for the determination of deoxynivalenol in wheat. Chromatographia 69: 1457-1462.

Prelusky, D.B., Rotter, B.A. and Rotter, R.G., 1994. Toxicology of mycotoxins. In: Miller, J.D. and Trenholm, H.L. (ed.) Mycotoxins in grain. Eagan Press, St. Paul, MN, USA, pp. 359-404.

Ran, R., Wang, C., Han, Z., Wu, A., Zhang, D. and Shi J., 2013. Determination of deoxynivalenol (DON) and its derivates: current status of analytical methods. Food Control 34: 138-148.

Ruan, R., Li, Y., Lin, X. and Chen, P., 2002. Non-destructive determination of deoxynivalenol levels in barley using near-infrared spectroscopy. Applied Engineering in Agriculture 18: 549-553.

Schothorst, R.C. and Jekel, A.A., 2001. Determination of trichothecenes in wheat by capillary gas chromatography with flame ionization detection. Food Chemistry 73: 111-117.

Sebaei, A.S., Gomaa, A.M., Mohamed, G.G. and Nour El-Dien, F.A., 2012. Simple validated method for determination of deoxynivalenol and zearalenone in some cereals using high performance liquid chromatography. American Journal of Food Technology 7: 668-678.

Shewry, P.R., 2009. Wheat. Journal of Experimental Botany 60: 1537-1553.

Sobrova, P., Adam, V., Vasatkova, A., Beklova, M., Zeman, L. and Kizek, R., 2010. Deoxynivalenol and its toxicity. Interdisciplinary Toxicology 3: 94-99.

Tanaka, T., Yoneda, A., Inoue, S., Sugiura, Y. and Ueno, Y., 2000. Simultaneous determination of trichothecene mycotoxins and zearalenone in cereals by gas chromatography-mass spectrometry. Journal of Chromatography A 882: 23-28.

Tangni, E., Motte, J.C., Callebaut, A. and Pussemier, L., 2010. Crossreactivity of antibodies in some commercial deoxynivalenol test kits against some fusariotoxins. Journal of Agricultural and Food Chemistry 58: 12625-12633.

Tralamazza, S.M., Bemvenuti, R.H., Zorzete, P., De Souza Garcia, F. and Corrêa, B., 2016. Fungal diversity and natural occurrence of deoxynivalenol and zearalenone in freshly harvested wheat grains from Brazil. Food Chemistry 196: 445-450.

Trucksess, M.W., Bao, L., Weaver, C.M. and White, K.D., 2010. Determination of deoxynivalenol in processed foods. Journal of AOAC International 93: 1236-1242.

Van der Fels-Klerx, H.J. and De Rijk, T.C., 2014. Performance evaluation of lateral flow immuno assay test kits for quantification of deoxynivalenol in wheat. Food Control 46: 390-396.

Vega, M. and Castillo, D., 2006. Determination of deoxynivalenol in wheat by validated GC/ECD method: comparison with HPTLC/ FLD. Electronic Journal of Food Plants Chemistry 1: 16-20. 